\title{
Photoelectrocatalytical degradation of basic blue 41 dye using nanoporous semiconductor of $\mathrm{Ti} / \mathrm{TiO}_{2}$
}

\author{
L. E.Fraga ${ }^{a}$, M. V. B. Zanoni ${ }^{a^{*}}$ \\ ${ }^{a}$ Departamento de Química Analítica, Instituto de Química, Universidade Estadual Paulista, Av. Prof. Francisco Degni, s/n, C.P. 355 , \\ 14801-970 Araraquara, SP, Brasil \\ "Autor correspondente: Tel. $+55-1633016600-6619$ \\ "E-mail:Boldrinv@iq.unesp.br
}

Resumo: O presente trabalho investiga a degradação fotoeletrocatalítica do corante Azul Básico 41 (AB 41) amplamente utilizado na tintura de fibras sintéticas, utilizando um semicondutor $\mathrm{Ti} / \mathrm{TiO}_{2}$ como fotoanodo. $100 \%$ de degradação foi obtida após $60 \mathrm{~min}$ de tratamento de $8,33 \times 10^{-}$ ${ }^{5} \mathrm{~mol} \mathrm{~L}^{-1}$ do corante em $0,1 \mathrm{~mol} \mathrm{~L}^{-1} \mathrm{Na}_{2} \mathrm{SO}_{4}$, pH 2 sob densidade de corrente de $0,40 \mathrm{~mA} \mathrm{~cm}^{-2} \mathrm{e}$ irradiação UV. Ainda foi obtido $80 \%$ de remoção de carbono orgânico total, cuja oxidação segue uma reação de pseudo-primeira ordem com constante de velocidade inicial de $-0,040 \mathrm{mim}^{-1}$ e uma eficiência de corrente de $51 \%$. Os resultados são superiores á fotocatálise convencional nas mesmas condições sem a polarização do fotoanodo que leva a $65 \%$ de mineralização sob constante de velocidade de $-0,024 \mathrm{mim}^{-1}$.

Palavras-chaves: corante azul básico 41 , fotoeletrocatálise, fotocatálise, $\mathrm{Ti} / \mathrm{TiO}_{2}$, tratamento de corantes.

\section{Introdução}

As indústrias têxteis lançam para os efluentes cerca de $10-15 \%$ de corantes que não são fixados nos substratos durante o processo de tingimento, podendo ser nocivo ao meio ao qual é aportado, uma vez que, alguns corantes utilizados nas indústrias têxteis, e seus subprodutos de biodegradação, podem apresentar alto grau de toxicidade, como mutagenicidade e carcinogenicidade ao ser humano[1-4].

O corante Azul Básico 41 (AB 41), Figura 1, ostenta um grupo cromóforo azo e, é utilizado na tintura de fibras sintéticas, tais como poliamida, poliéster e viscose[5,6]. Corantes básicos (catiônicos) que, em sua maioria, apresentam estabilidade à hidrólise, são sensíveis ao $\mathrm{pH}$ e solúveis em meio aquoso[7]. Portanto requerem métodos de tratamento mais eficientes para sua completa remoção em águas superficiais e efluentes.<smiles>CCN(CCO)c1ccc(N=Nc2nc3ccc(OC)cc3s2)cc1</smiles>

Figura 1: Estrutura do corante azul básico 41.

A técnica de fotoeletrocatálise utilizando semicondutores como $\mathrm{TiO}_{2}, \mathrm{WO}_{3}, \mathrm{ZnO}, \mathrm{SnO}_{2}$, entre outros, são reportados na literatura como uma alternativa promissora no tratamento de corantes, promovendo simultaneamente a descoloração e mineralização do poluente orgânico[3,8-10]. As reações fotocatalíticas sobre superfícies semicondutoras se processam segundo as etapas básicas de: excitação com luz de energia maior que a banda de energia proibida, "band gap" $\left(\mathrm{E}_{\mathrm{g}}\right)$, do 
semicondutor, geração de pares de elétron/lacuna $\left(\mathrm{e}^{-} / \mathrm{h}^{+}\right)$; aprisionando elétrons e lacunas pelas espécies adsorvidas. O mecanismo de processos redox mediado por semicondutores do tipo n como $\mathrm{TiO}_{2}$ e $\mathrm{WO}_{3}$ em meio aquoso promove a formação do radical hidroxila $\left(\mathrm{OH}^{\circ}\right)$, poderoso agente oxidante $(+2,72 \mathrm{~V} v s$. ENH $)$, gerado pela promoção da oxidação da água/hidroxilas adsorvidas pela lacuna, tornando o processo fotocatalítico altamente eficiente para oxidar a maioria dos compostos orgânicos[11-13]. Considerando que a eficiência desse processo esta relacionada ao tempo de vida das lacunas $\left(\mathrm{h}^{+}{ }_{\text {bv }}\right)$ e retardamento da velocidade de recombinação das cargas geradas $\left(\mathrm{e}^{-} / \mathrm{h}^{+}\right)$no semicondutor, a fotoeletrocatálise tem sido uma metodologia difundida para a ampliação na eficiência, com a aplicação de um potencial ou densidade de corrente externo. Quando a densidade de corrente é aplicada ao semicondutor, há a formação de um gradiente de densidade de corrente que impulsiona o elétron para o contra eletrodo e a lacuna para a superfície do eletrodo, diminuindo a velocidade de recombinação, promovendo um consequente aumento da eficiência fotoeletrocatalítica com a geração de radicais hidroxilas, conforme as equações abaixo[14]:

$$
\begin{aligned}
& \text { Fotoânodo: } \begin{array}{l}
\mathrm{TiO}_{2}+h v \rightarrow \mathrm{TiO}_{2}-\mathrm{e}_{\mathrm{bc}}^{-}+\mathrm{TiO}_{2}- \\
\mathrm{h}^{+}{ }_{\mathrm{bv}}
\end{array} \\
& \mathrm{TiO}_{2}-\mathrm{h}_{\text {bv }}^{+}+\mathrm{H}_{2} \mathrm{O}_{\text {ads }} \rightarrow \mathrm{TiO}_{2}-\mathrm{OH}_{\mathrm{s}}^{\cdot}+\mathrm{H}^{+} 2 \\
& \text { Cátodo: } \quad \mathrm{H}_{2} \mathrm{O}+2 \mathrm{e}^{-} \rightarrow \mathrm{H}_{2}+2 \mathrm{OH}^{-}
\end{aligned}
$$

No entanto a maioria dos trabalhos para tratamento fotoeletrocatalítico de corantes descritos na literatura [15-17], são baseados para corantes do tipo disperso, reativo e ácidos, e os trabalhos relacionados com corantes básicos são raros. Deste modo, considerando que cada grupo de corantes pertence a classes distintas com estruturas e propriedades físico-químicas diferentes, é objetivo do presente trabalho investigar a degradação fotoeletrocatalítica do corante azul básico 41 utilizando semicondutor nanoporoso de $\mathrm{Ti} / \mathrm{TiO}_{2} \mathrm{em}$ um reator fotoeletroquímico usando irradição UV, visando desenvolver um método alternativo para remoção de corantes do tipo básico.

\section{Parte Experimental}

a) Preparação do semicondutor nanoporoso de $\mathrm{Ti} / \mathrm{TiO} 2$

O semicondutor foi preparado segundo procedimento previamente descrito na literatura[3,18]. uma placa de titânio (área de $25 \mathrm{~cm}^{2}$ ) foi lavada, imersa em acetona e submetida à ultrasson durante cinco horas. Em seguida foi calcinada por três horas sob temperatura de $350{ }^{\circ} \mathrm{C}$. A placa foi recoberta com dióxido de titânio em suspensão através da técnica de Dip-coating, seca em estufa por uma hora a $100{ }^{\circ} \mathrm{C}$ e, em seguida calcinada a $350{ }^{\circ} \mathrm{C}$ por três horas. Este procedimento foi repetido por mais quatro vezes. As suspensões de $\mathrm{TiO}_{2}$ foram preparadas pelo processo sol-gel, utilizando tetraisopropóxido de titânio e ácido nítrico nas razões $\mathrm{Ti} / \mathrm{H}^{+} / \mathrm{H}_{2} \mathrm{O}$ de $1 / 0,5 / 200 \mathrm{~mL}$, submetido à processo de diálise até pH em 3,5.[11,19].

\section{b) Instrumental}

As medidas de $\mathrm{pH}$ foram realizadas em pHmetro Corning modelo 455. Nas medidas de densidade de corrente constante, utilizou-se um galvanostato PAR 283. As medidas espectrofotométricas na região do UV-vis foram realizadas em um espectrofotômetro Hewlett Packard modelo 8453 interfaciado por um programa UV- visible Chemstation Software Hp-854X, com cubetas de quartzo de $1 \mathrm{~cm}$ de caminho óptico. As análises de carbono orgânico total (COT) foram realizadas com um analisador de carbono TOC-Vcpn com injetor automático ASI-V - Shimadzu.

\section{c) Oxidação Fotoeletrocatalítica}

Os experimentos de fotoeletrocatálise e fotocatálise do corante azul básico 41 foram realizados em um reator desenvolvido no laboratório de Eletroanalítica no Instituto de Química da UNESP-Araraquara. Este reator é composto de uma célula de quartzo com capacidade de $250 \mathrm{~mL}$, com dois eletrodos (trabalho: eletrodo nanoporoso de $\mathrm{Ti} / \mathrm{TiO}_{2}$ e contra-eletrodo: rede de platina), 
um tubo de quartzo para a imersão da lâmpada de vapor de mercúrio $125 \mathrm{~W}$ e um borbulhador de ar. As medidas foram realizadas em sulfato de sódio $0,10 \mathrm{~mol} \mathrm{~L}^{-1}$ e $8,33 \times 10^{-5} \mathrm{~mol} \mathrm{~L}^{-1}$ do corante azul básico 41 . Alíquotas de $2,0 \mathrm{~mL}$ foram removidas sob tempo controlado e submetidas à análise de UVVis e análise de carbono orgânico total.
Voltamogramas lineares de fotocorrente $v s$ potencial foram obtidos no intervalo de $-550 \mathrm{a}$ $1500 \mathrm{mV}$, a $10 \mathrm{mv} \mathrm{s}^{-1}$ para o eletrodo de $\mathrm{Ti} / \mathrm{TiO}_{2}$ em presença de $\mathrm{Na}_{2} \mathrm{SO}_{4} 0,1 \mathrm{~mol} \mathrm{~L}^{-1}$, como mostra a Figura 2.

\section{Resultados e Discussão}

a) Fotoatividade do semicondutor nanoporoso de $\mathrm{Ti} / \mathrm{TiO}_{2}$

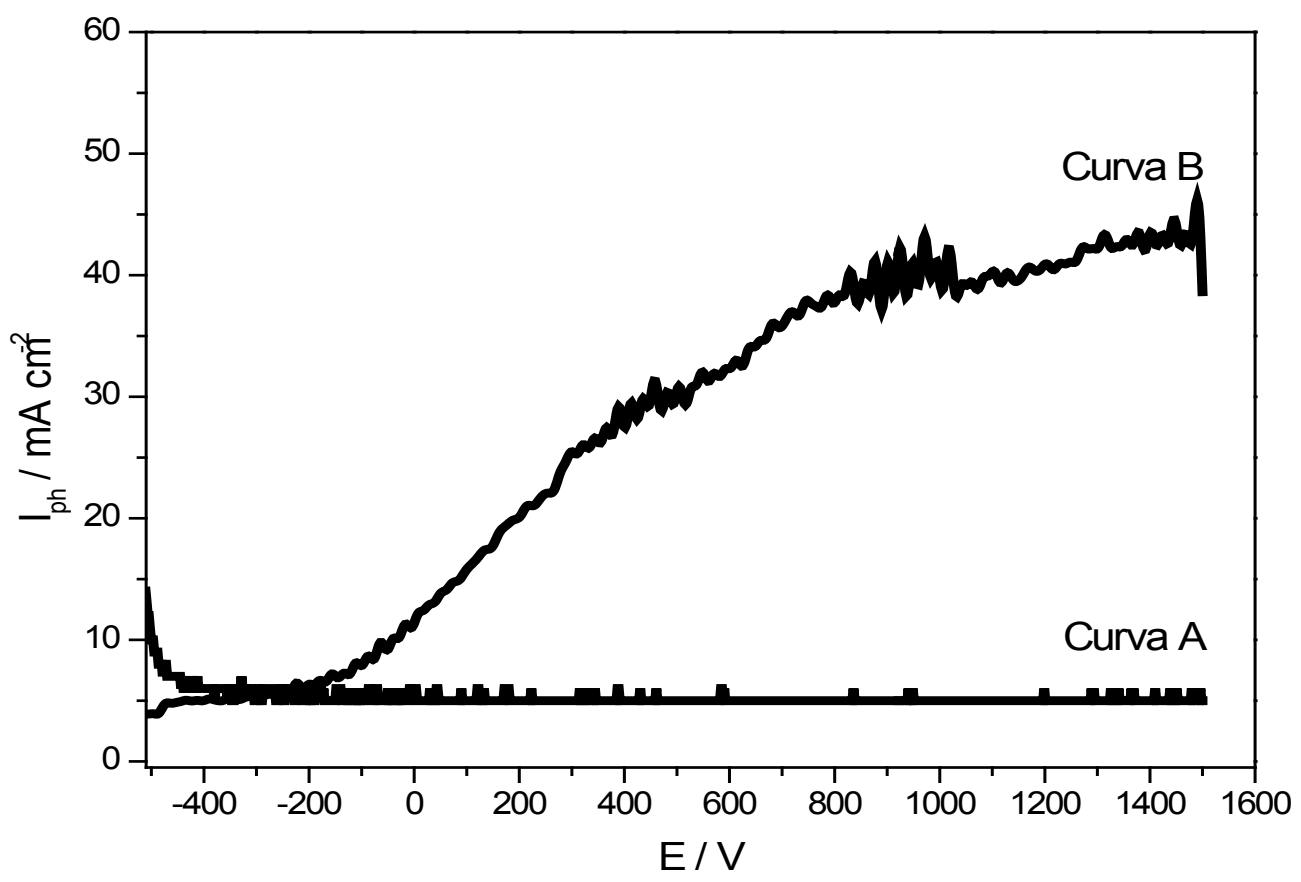

Figura 2 - Curvas de fotocorrente-potencial obtidas para o semicondutor nanoporoso de $\mathrm{Ti} / \mathrm{TiO}_{2}$ sem irradiação (Curva a), sob irradiação UV em Na $\mathrm{SO}_{4} 0,10 \mathrm{~mol} \mathrm{~L}^{-1}$ (Curva b), $\mathrm{pH}=2,0$ e $v \stackrel{2}{=}$ $0,01 \mathrm{~V} \mathrm{~s}^{-1}$.

A fotocorrente é negligenciável sob condições sem irradiação UV (curva A). Entretanto, sob irradiação (curva B), há um aumento da fotocorrente em potenciais acima de $-200 \mathrm{mV}$, indicando que o par $\mathrm{e}^{-} / \mathrm{h}^{+}$é gerado quando o semicondutor é irradiado por luz UV $(\mathrm{h} v)$ com energia maior que a energia da banda proibida deste 
semicondutor, e há uma favorável separação das cargas fotogeradas, sob polarização do eletrodo num potencial mais positivo que o potencial de banda plana (flat band), simultaneamente a irradiação UV, assim observando um gradiente de densidade de corrente impulsionado as lacunas para a superfície do semicondutor e os elétrons para o interior, que são levados ao contra-eletrodo não fotoatico[20]. Este processo diminui a recombinação $\mathrm{e}^{-} / \mathrm{h}^{+}$aumentando o tempo de vida dos radicais $\mathrm{OH}^{*}$ gerados na superfície do eletrodo de trabalho e como conseqüência na melhor eficiência do processo.

\section{b) Oxidação fotoeletrocatalítica corante do azul} básico 41

\section{b.1) Efeito da variação do $\mathrm{pH}$}

A oxidação fotoeletrocatalítica de $8,33 \times 10^{-}$ ${ }^{5} \mathrm{~mol} \mathrm{~L}^{-1}$ do corante azul básico 41 em $\mathrm{Na}_{2} \mathrm{SO}_{4} 0,1$ mol L $\mathrm{L}^{-1}$ foi investigada em $\mathrm{pH}$ inicial de $2,4,6 \mathrm{e}$ 8. Os respectivos valores obtidos para a descoloração do corante $(\lambda=590 \mathrm{~nm})$ são mostrados na Figura 3.

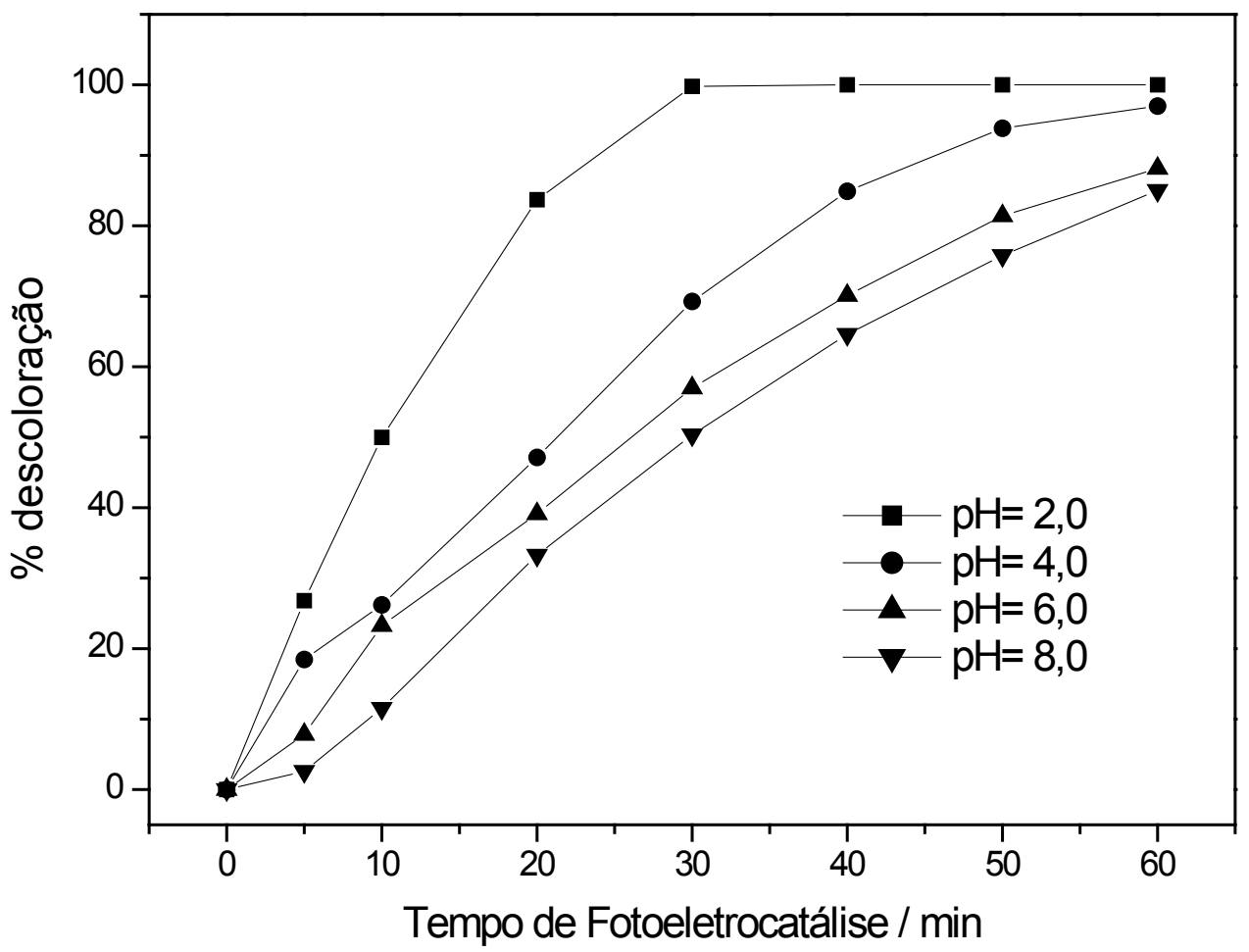

Figura 3 - Porcentagem de descoloração $\left(\lambda_{\max }=590 \mathrm{~nm}\right)$ do [azul 41] $=8,33 \times 10^{-5} \mathrm{~mol} \mathrm{~L}^{-1} \mathrm{em} \mathrm{Na}_{2} \mathrm{SO}_{4} 0,10$ mol L-1, utilizando eletrodo nanoporoso de $\mathrm{Ti}_{\text {i }} / \mathrm{TiO}_{2}$, sob irradiação UV. 
Observa-se que o tratamento fotoeletroquímico promove valores entre $85 \%$ a $100 \%$ de descoloração nos valores de $\mathrm{pH}$ estudados. No entanto o processo é mais eficiente em $\mathrm{pH}$ 2, quando atinge $100 \%$ de descoloração após 30 minutos de oxidação.

De acordo com a literatura, sabendo-se que o estudo do potencial de carga zero[21] do semicondutor de $\mathrm{Ti} / \mathrm{TiO}_{2}$, é ao redor de $\mathrm{pH} \mathrm{5,} \mathrm{nesta}$ região, a superfície do semicondutor encontra-se carregado positivamente, e a solução do corante está carregada negativamente, uma vez que possui pka $\leq 1,2$; facilitando a adsorção do corante na superfície do semicondutor, aumentando consequentemente a eficiência do processo.

\section{b.2) Efeito da densidade de corrente}

No intuito de melhorar a eficiência da degradação fotoeletrocatalítica, com semicondutor nanoporoso de $\mathrm{Ti} / \mathrm{TiO}_{2}$, do corante azul básico 41 , estudamos o efeito da densidade de corrente na eficiência do processo. As densidades de correntes aplicadas foram 0,$20 ; 0,40 ; 0,60$ e $0,80 \mathrm{~mA}$ $\mathrm{cm}^{-2}$. Para isto, utilizou-se $8,33 \times 10^{-5} \mathrm{~mol} \mathrm{~L}^{-1}$ do corante em $\mathrm{Na}_{2} \mathrm{SO}_{4} 0,10 \mathrm{~mol} \mathrm{~L}^{-1}, \mathrm{pH}=2,0$ e irradiação UV para valores de corrente entre 10, 20, 30 e $40 \mathrm{~mA}$.

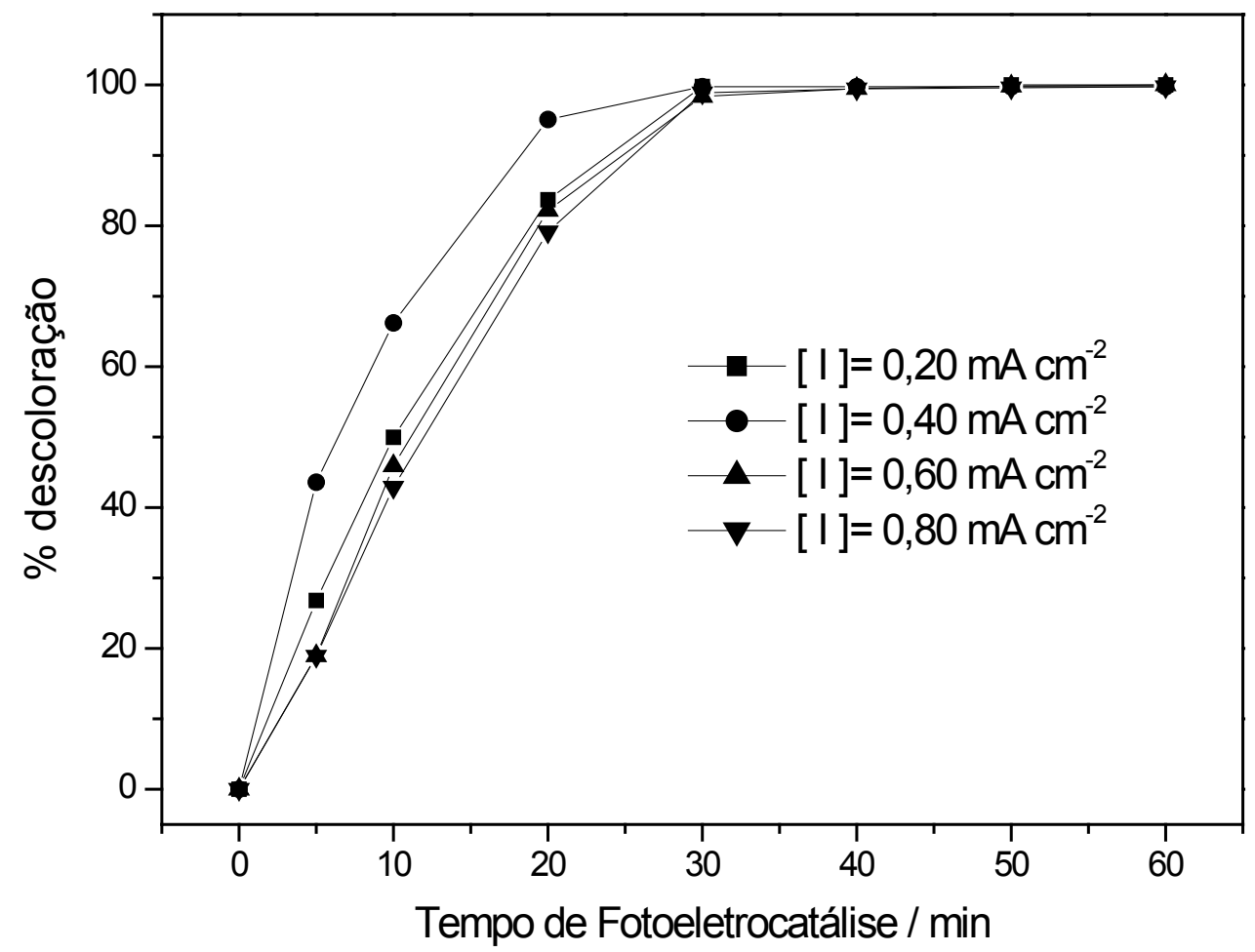

Figura 4 - Porcentagem de descoloração $\left(\lambda_{\max }=590 \mathrm{~nm}\right)$ do [azul 41] $=8,33 \times 10^{-5} \mathrm{~mol} \mathrm{~L}^{-1} \mathrm{em} \mathrm{Na}_{2} \mathrm{SO}_{4} 0,10$ mol L-1 ${ }^{-1} \mathrm{pH}=2,0$ e sob irradiação UV.

A figura 4 mostra o efeito da densidade de corrente na descoloração do corante. Para todos os valores de densidade de corrente aplicados, há $100 \%$ de descoloração da solução. Entretanto sob densidade de corrente de 0,40 $\mathrm{mA} \mathrm{cm}^{-2}$, observa-se maior eficiência do processo, alcançando $100 \%$ de remoção da cor em apenas 20 minutos de tratamento. 
A eficiência de corrente foi calculada usando a seguinte relação, de acordo com a literatu$\mathrm{ra}[22,23]$.

$$
\mathrm{EC}(\%)=\frac{\left[(\mathrm{COT})_{0}-(\mathrm{COT})_{\mathrm{t}}\right.}{8 \mathrm{I} \Delta \mathrm{t}} \mathrm{FV} 100
$$

tal $\left(\mathrm{g} \mathrm{L}^{-1}\right)$ nos tempos 0 e $\mathrm{t}$, respectivamente, $\mathrm{I}$ a corrente (A), F a constante faradaica (26,8 Ah), V o volume da solução (L) e $\Delta$ t o tempo de tratamento (h). Obteve-se $51 \%$ de eficiência de corrente nas condições fotoeletrocatalíticas otimizadas em 40 minutos de tratamento, Figura 5.

Onde $(\mathrm{COT})_{0}$ e (TOC) $)_{\mathrm{t}}$ são o carbono orgânico to-

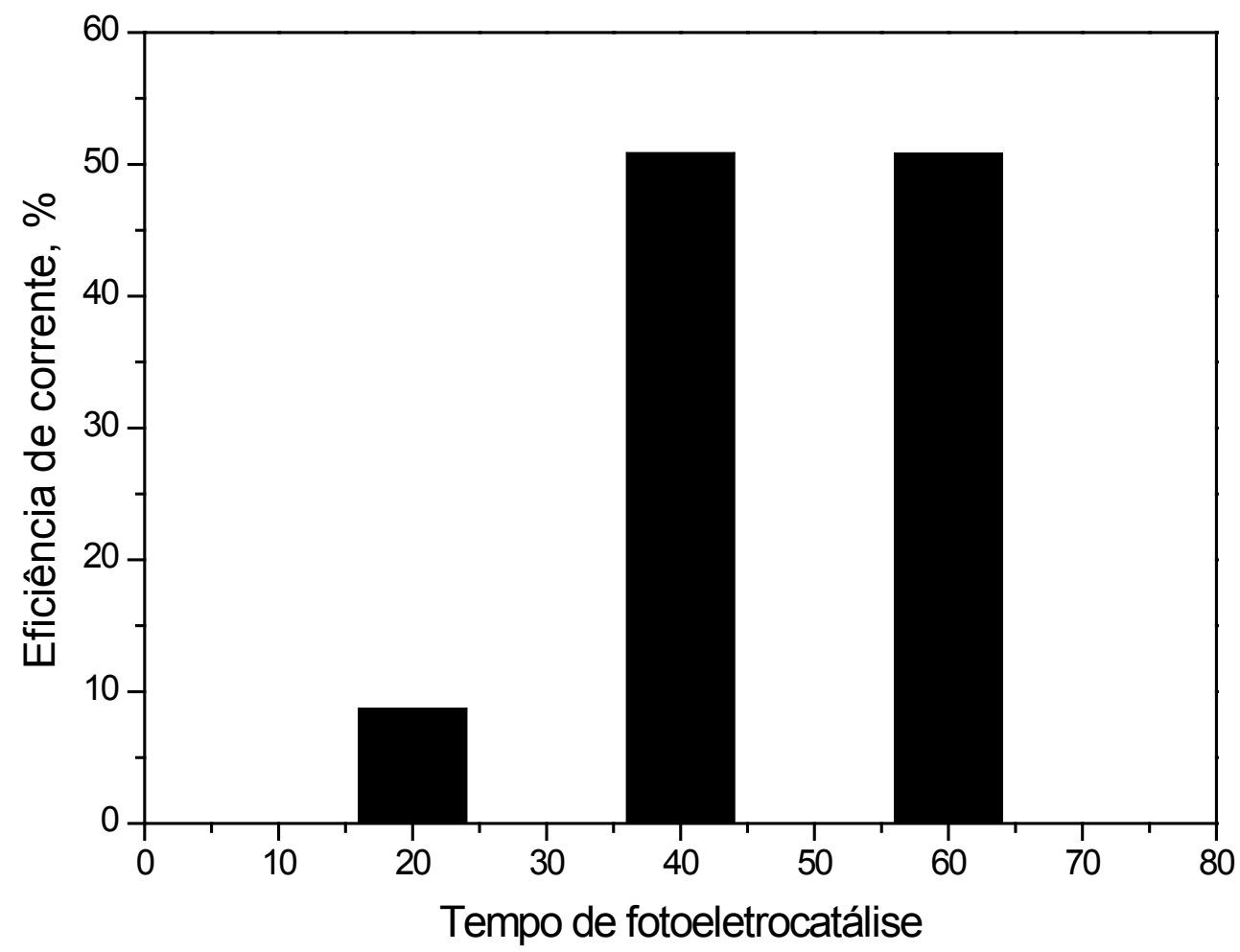

Figura 5 - Eficiência de corrente em função do tempo de fotoeletrocatálise (20, 40 e 60 minutos) sob aplicação de densidade de corrente de $0,40 \mathrm{~mA} \mathrm{~cm}^{-2}$.

\section{a) Avaliação da mineralização do corante azul básico 41}

A mineralização do composto foi monitorada por medidas de carbono orgânico total (COT), seguindo análises da alíquotas removidas durante 60 minutos de tratamento.

A porcentagem de degradação do corante azul básico 41 foi obtida pela equação: \% de degradação de $\mathrm{COT}=\left[\left(\mathrm{COT}_{\text {inicial }}-\mathrm{COT}_{\text {final }}\right) / \mathrm{COT}_{\text {inicial }}\right] \times 100$, nas condições otimizadas de fotoeletrocatálise: $\mathrm{pH}=$ 2,0; densidade de corrente $0,40 \mathrm{~mA} \mathrm{~cm}^{-2}, \mathrm{Na}_{2} \mathrm{SO}_{4} 0,10 \mathrm{~mol} \mathrm{~L}^{-1}$, sob irradiação UV e $8,33 \times 10^{-5} \mathrm{~mol} \mathrm{~L}^{-1}$ do corante. O resultados são apresentados na Figura 6. 


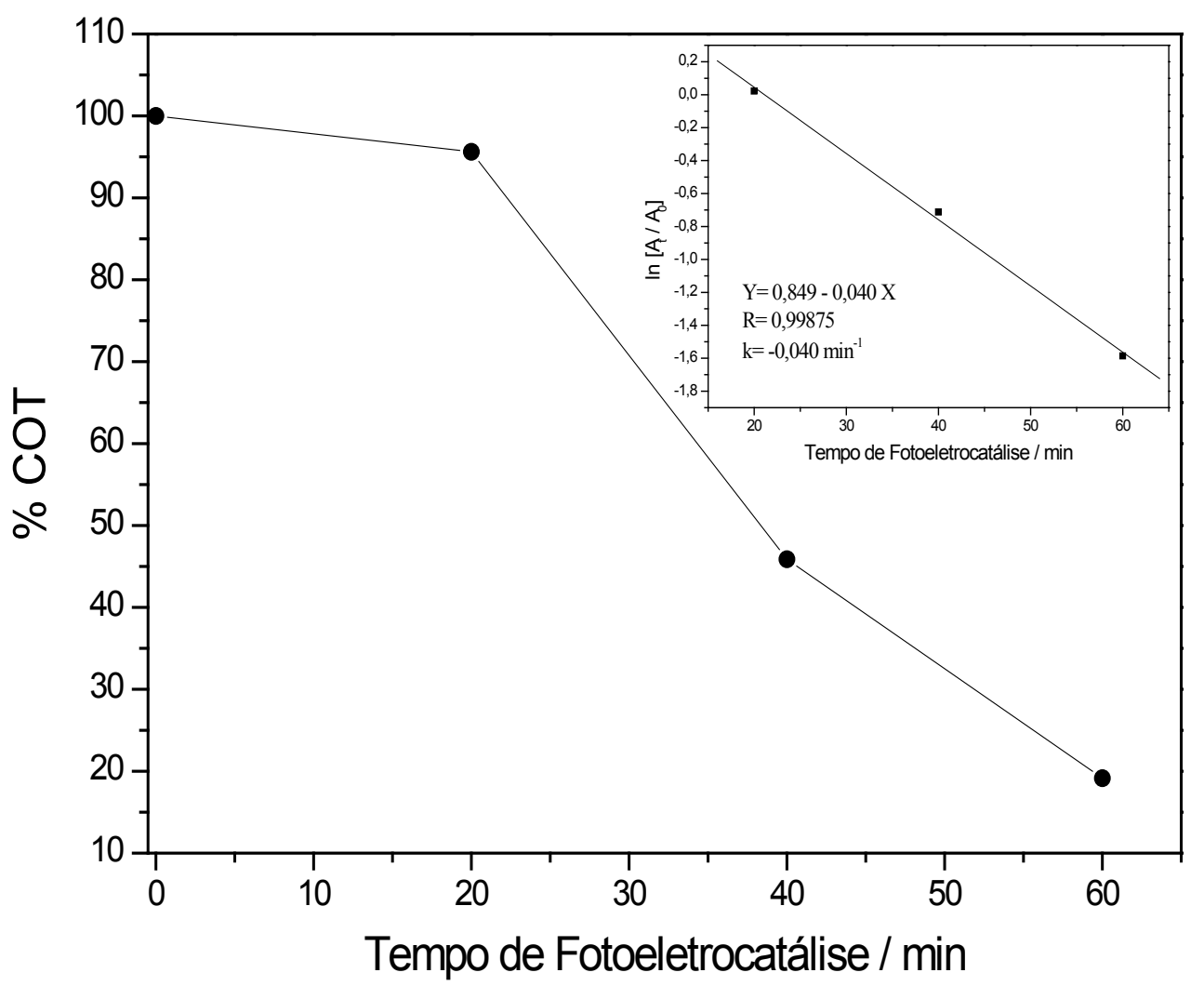

Figura 6 - Porcentagem de degradação do COT do azul 41 para as condições de fotoeletrocatálise otimizadas: $\mathrm{pH}=2,0$; [ I ] = 0,40 mA cm${ }^{-2}, \mathrm{Na}_{2} \mathrm{SO}_{4} 0,10 \mathrm{~mol} \mathrm{~L}^{-1}$, [azul 41] $=8,33 \times 10^{-5} \mathrm{~mol} \mathrm{~L}^{-1}$ e sob irradiação UV.

Degradação de $80 \%$ do corante é obtida após 60 minutos de fotoeletrocatálise. A cinética de degradação do corante azul 41 foi investigada usando a relação $\ln [\mathrm{A}]_{\mathrm{t}} /[\mathrm{A}]_{0}$ vs tempo de fotoeletrocatálise, sendo []$_{\mathrm{t}} \mathrm{e}[]_{0}$ a concentração da matéria orgânica remanescente no tempo $t$ e no tempo zero, usando medidas de COT, e observou um decaimento segundo uma cinética de pseudoprimeira ordem, cujo valor da constante de velocidade $(\mathrm{k})$ de degradação fotoeletrocatalítica do COT é de $-0,040 \mathrm{~min}^{-1}$.

\section{b) Comparação entre oxidação fotoeletrocatalíti- ca e fotocatalítica}

Os processos fotoeletrocatalítico e fotocatalítico foram comparados usando as condições otimizadas de $\mathrm{pH}=2,0 ; \mathrm{Na}_{2} \mathrm{SO}_{4} 0,10 \mathrm{~mol} \mathrm{~L}^{-1}$, concentração do corante $8,33 \times 10^{-5} \mathrm{~mol} \mathrm{~L}^{-1}$ e densidade de corrente $0,40 \mathrm{~mA} \mathrm{~cm}^{-2}$ (fotoeletrocatálise), sob irradiação UV. A partir dos valores de absorbância fixos em $\lambda_{\max }=590 \mathrm{~nm}$, observou-se a descoloração do corante $\mathrm{AB} 41$, em ambos os processos, como mostra a Figura 7. Remoção de $100 \%$ da cor é obtida após 60 minutos de tratamento, para ambos os processos. Os valores da constante de velocidade (k) da descoloração fotoeletrocatalítica e fotocatalítica são $\mathrm{k}=-0,11566 \mathrm{~min}^{-1}$ e $\mathrm{k}=-0,05253$ $\mathrm{min}^{-1}$, segundo a cinética de pseudo-primeira ordem, Figura 8. 


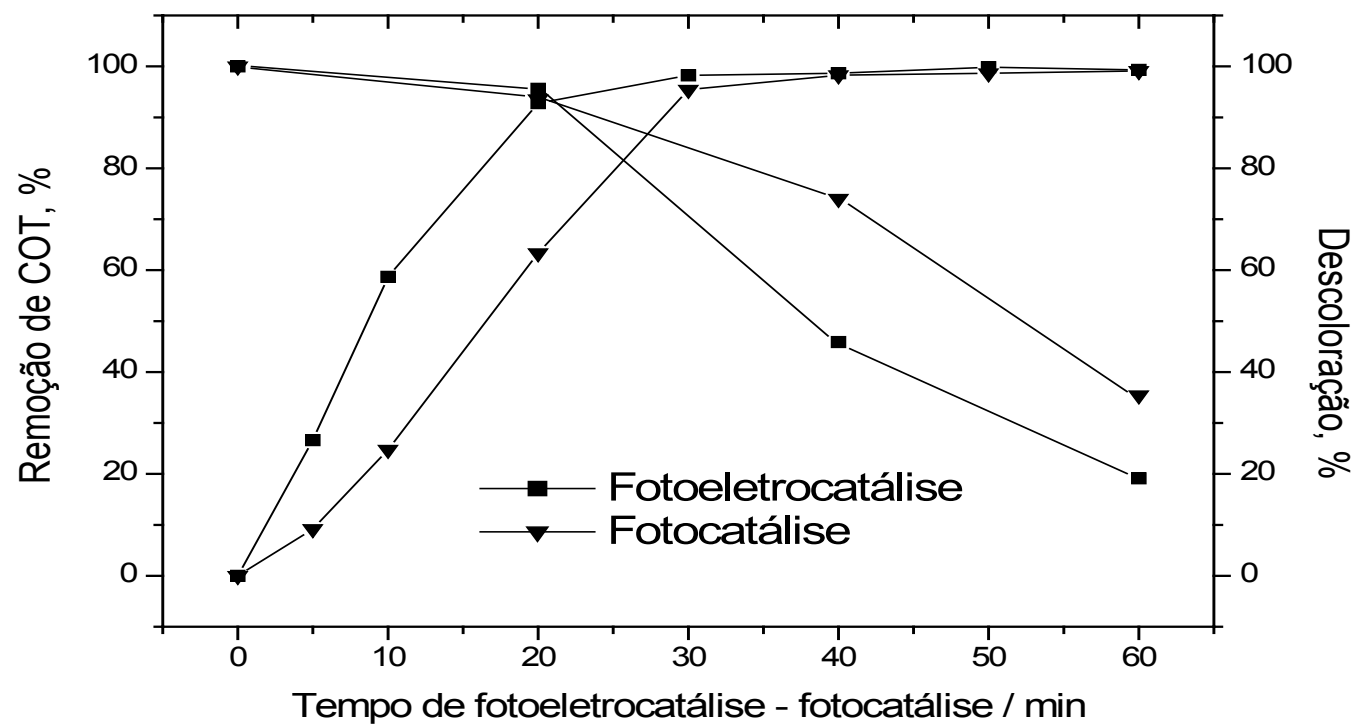

Figura 7 - Porcentagem de descoloração, em $\lambda_{\max }=590 \mathrm{~nm}$, e remoção de COT do corante azul básico 41, através da fotoeletrocatálise ([ I ] $\left.=0,40 \mathrm{~mA} \mathrm{~cm}^{-2}\right)$ e fotocatálise, em $\mathrm{pH}=2,0 ; \mathrm{Na}_{2} \mathrm{SO}_{4} 0,10 \mathrm{~mol} \mathrm{~L}^{-1}$ e sob irradiação UV.

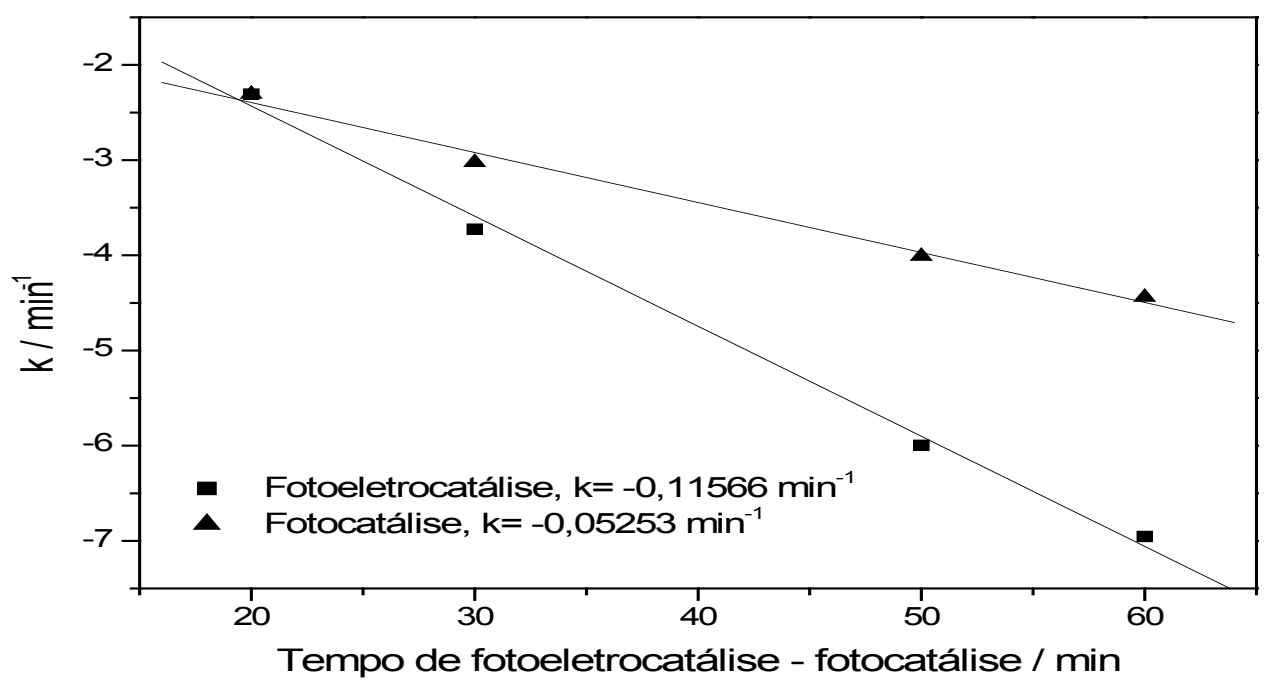

Figura 8 - Gráfico de $\ln \left[\mathrm{A}_{\mathrm{t}} / \mathrm{A}_{0}\right]$ vs tempo de fotoeletrocatálise $\left(0,40 \mathrm{~mA} \mathrm{~cm}{ }^{-2}\right)$ e fotocatálise na descoloração do corante azul básico 41, em pH=2,0; [ I ] $=0,40 \mathrm{~mA} \mathrm{~cm}^{-2}, \mathrm{Na}_{2} \mathrm{SO}_{4} 0,10 \mathrm{~mol} \mathrm{~L}^{-1}$ e sob irradiação UV. 
No entanto, o estudo de degradação na remoção do COT nos processos fotoeletrocatalítico e fotocatalítico do azul básico 41 são diferentes como apresentado também na Figura 7. Observase a degradação de $81 \%$ de COT no processo fotoeletrocatalítico e apenas $65 \%$ de COT na fotocatálise.

A cinética de degradação do azul básico 41 foi determinada através da relação $\ln [\mathrm{A}]_{\mathrm{t}} /[\mathrm{A}]_{0} v s$ tempo de fotoeletrocatálise, tomando-se para tal a degradação do carbono orgânico total do corante azul básico 41. A partir da Figura 9, determinouse o valor da constante de velocidade $(\mathrm{k})$ da degradação fotocatalítica do corante azul básico 41, que é $\mathrm{k}=-0,024 \mathrm{~min}^{-1}$, confirmando assim a maior eficiência do processo fotoeletrocatalítico em relação ao fotocatalítico, que apresenta valores de $0,040 \mathrm{~min}^{-1}$.

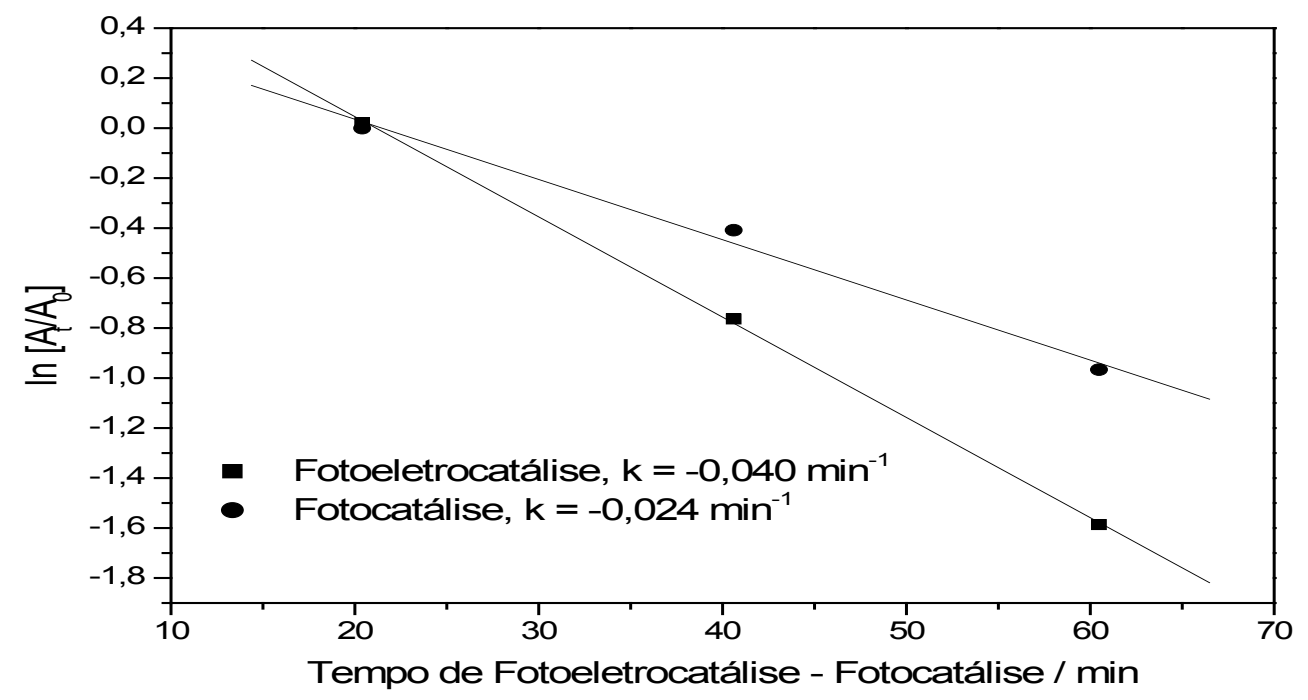

Figura 9 - Gráfico de $\ln \left[\mathrm{A}_{t} / \mathrm{A}_{0}\right]$ vs tempo de fotoeletrocatálise e fotocatálise na degradação de COT do corante azul básico 41, em pH=2,0; [ I ] = 0,40 mA cm${ }^{-2}, \mathrm{Na}_{2} \mathrm{SO}_{4} 0,10 \mathrm{~mol} \mathrm{~L}^{-1}$ e sob irradiação UV.

Portanto, o processo fotoeletrocatalítico apresentou uma maior performance em relação à fotocatálise, tanto na descoloração do azul 41 como na mineralização do composto, evidenciando a eficiência da densidade de corrente sobre o processo fotocatalítico.

\section{Conclusão}

O semicondutor nanoporoso de $\mathrm{Ti} / \mathrm{TiO}_{2}$ apresenta uma boa fotoatividade, com valores de fotocorrente ao redor de $40 \mathrm{~mA} \mathrm{~cm} \mathrm{~cm}^{-2}$ potencial de $1000 \mathrm{mV}$ e pode ser usado como fotoanodo nas medidas de oxidação fotoeletrocatalítica. A melhores condições experimentais para a degradação do corante azul básico 41 é em meio ácido ( $\mathrm{pH}=$ $2,0)$, densidade de corrente igual a $0,40 \mathrm{~mA} \mathrm{~cm}^{-2}$. Usando as melhores condições experimentais, o processo fotoeletrocatalítico sob irradiação UV, promoveu a descoloração de $100 \%$ da solução e remoção de $81 \%$ de COT após 60 minutos sob constante de velocidade de degradação inicial de $-0,040 \mathrm{mim}^{-1}$ e uma eficiência de corrente de $51 \%$ em 40 minutos de tratamento. $\mathrm{O}$ processo mostrou-se mais eficiente quando comparado à fotocatálise, que nas mesmas condições experimentais apresenta constante de degradação na ordem de 
$0,024 \mathrm{~min}^{-2}$. Deste modo, os resultados ilustram que o processo fotoeletrocatalítico, utilizando semicondutor nanoporoso de $\mathrm{Ti} / \mathrm{TiO}_{2}$ como fotoanodo, pode ser aplicado como uma técnica alternativa na remoção de corantes básicos.

\section{Agradecimentos}

Os autores agradecem a FAPESP e a CAPES pelos financiamentos.

\begin{abstract}
The present work investigates the photoelectrocatalytical degradation Basic Blue 41 (BB 41) largely applied to dye synthetic fibers, using a semiconductor $\mathrm{Ti} / \mathrm{TiO}_{2}$ as photoanode. $100 \%$ of degradation of $8.3310^{-5} \mathrm{~mol} \mathrm{~L}^{-1}$ dye in $0.1 \mathrm{~mol} \mathrm{~L}^{-1} \mathrm{Na}_{2} \mathrm{SO}_{4}$, $\mathrm{pH} 2$ was obtained under current density of $0.40 \mathrm{~mA} \mathrm{~cm}{ }^{-2}$ and irradiation UV after 60 min of treatment and $80 \%$ of total organic carbon removal. The oxidation follows pseudo-first order reaction with initial rate constant of $-0,040 \mathrm{mim}^{-1}$ and a current efficiency of $51 \%$. The results are superior the conventional photocatalysis in the same conditions without the polarization of the photoanode that takes to $65 \%$ of mineralization under initial rate constant $-0,024 \mathrm{~min}^{-1}$.
\end{abstract}

Keywords: basic blue 41, photoelectricatalysis, photocatalysis, $\mathrm{Ti} / \mathrm{TiO}_{2}$, dye treatment.

\section{Referências}

[1] - C. Fernández, M. L. Larrechi, M. P. Callao, Talanta, 79 (2009) 1292-1297.

[2] - R. M. Liversidge, G. J. Lloyd, D. A. J. Wase, C. F. Forster, Process Biochemistry, 32 (1997) 473-477.

[3] - P. A. Carneiro, M. E. Osugi, J. J. Sene, M. A. Anderson, M. V. B. Zanoni, Electrochimica Acta, 49 (2004) 3807-3820. [4] - Y. Jiang, Y. Sun, H. Liu, F. Zhu, H. Yin, Dyes and Pigments, 78 (2008) 77-83.

[5] - M. Roulia, A. A. Vassiliadis, J. Colloid Interface Sci, 291 (2005) 37-44.

[6] - M. Abbasi, N. R. Asl, Journal of Hazardous Materials, 153(2008) 942-947.

[7] - Barcellos, I. O. Química Têxtil II. Blumenau, 2004, $183 \mathrm{p}$.

[8] - C. A. M. Huitle, E. Brillas, Applied Catalysis B: Environmental, 87 (2009) 105-145.

[9] - Y. Xu, et al.. Chem. Eng. J. (2009), doi:10.1016/j. cej.2009.01.002.

[10] - L. C. Macedo, D. A. M. Zaia, G. J. Moore; H. Santana, Journal of Photochemistry and Photobiology A: Chemistry, 185 (2007) 86-93.

[11] - M. V. B. Zanoni, J. J. Sene, M. A. Anderson, Journal Photochemistry Photobiology A: Chemistry, 157 (2003) 5563.

[12] - M. E. Osugi, G. A. Umbuzeiro, M. A. Anderson, M. V. B. Zanoni, Electrochimica Acta,50 (2005) 5261-5269.

[13] - M. E. Osugi, G. A. Umbuzeiro, F. J. V. Castro, M. V. B. Zanoni, Journal of Hazardous Materials B, 137 (2006) 871877.
[14] - S. T. Martin, H. Herrmann, W. Choi, M. R. Hoffmann, Part 1., Trans. Faraday Soc., 91 (1994) 3315.

[15] - A. C. Patricia et al. Chemosphere, 59 (2005) 431-439.

[16] - F. H. Oliveira, M. E Osugi, F. M. M. Paschoal, D. Profeti, P. Olivi, M. V. B. Zanoni, Journal of Applied Electrochemistry, 37 (2007) 583-592.

[17] - M. E. Osugi, C. C. I. Guaratini, N. R. Stradiotto, M. V. B. Zanoni, Química Nova, 27(3) (2004) 417-420.

[18] - G. Patermarakis, E. Fountoukidus, Water Res. 24 (1990) 1491-1496.

[19] - L. E. Fraga, M. A. Anderson, M. L. P. M. A. Beatriz, F. M. M. Paschoal, Romão, L. P. Romão, M. V. B. Zanoni, Electrochimica Acta, 54 (2009) 2069-2076.

[20] - Blesa, M. A.. (Ed.). La Plata: Digital Grafic, 2001. 316 p.

[21] - Paschoal, Fabiana Maria Monteiro. Aplicações da técnica fotoeletrocatalítico na oxidação de corantes ácidos, corantes dispersos, surfatantes e na redução de $\mathrm{Cr}(\mathrm{VI})$ e bromato em efluentes usando eletrodos nanoporosos de $\mathrm{Ti} / \mathrm{TiO}_{2}$. Araraquara, 2008. Tese de Doutorado. Instituto de Química, UNESP.

[22] - C. R. Costa, C. M. R. Botta, E. L. G. Espindola, P. Olivi, Journal of Hazardous Materials 153 (2008) 616.

[23] - M.A. Butler, Journal Appl. Phys., 48 (1977) 1914. 